\author{
MITSUBISHI ELECTRIC RESEARCH LABORATORIES \\ http://www.merl.com
}

\title{
Decentralized Finite-Time Stabilizing Feedback Network Control
}

\author{
Zhao, Y.
}

TR2012-100 October 2012

\begin{abstract}
This work explores the stability properties of a class of nonlinear system, which is obtained by applying a special transformation to the vector field of a linear system. It is proved that such a nonlinear system preserves the stability of the original linear system. This property can be used for checking the stability of certain nonlinear systems, and designing novel network control protocols based on homogeneous system theory. For the network control application, finitetime convergence can be achieved in a decentralized manner by properly choosing the smooth feedback control law. The results are demonstrated by numerical examples.
\end{abstract}

ASME Dynamic Systems and Control Conference (DSCC)

This work may not be copied or reproduced in whole or in part for any commercial purpose. Permission to copy in whole or in part without payment of fee is granted for nonprofit educational and research purposes provided that all such whole or partial copies include the following: a notice that such copying is by permission of Mitsubishi Electric Research Laboratories, Inc.; an acknowledgment of the authors and individual contributions to the work; and all applicable portions of the copyright notice. Copying, reproduction, or republishing for any other purpose shall require a license with payment of fee to Mitsubishi Electric Research Laboratories, Inc. All rights reserved.

Copyright (C) Mitsubishi Electric Research Laboratories, Inc., 2012

201 Broadway, Cambridge, Massachusetts 02139 



\title{
DECENTRALIZED FINITE-TIME STABILIZING FEEDBACK NETWORK CONTROL
}

\author{
Yiming Zhao* \\ Mitsubishi Electric Research Laboratories \\ 201 Broadway, Cambridge, MA \\ Email: yzhao@merl.com
}

\begin{abstract}
This work explores the stability properties of a class of nonlinear system, which is obtained by applying a special transformation to the vector field of a linear system. It is proved that such a nonlinear system preserves the stability of the original linear system. This property can be used for checking the stability of certain nonlinear systems, and designing novel network control protocols based on homogeneous system theory. For the network control application, finite-time convergence can be achieved in a decentralized manner by properly choosing the smooth feedback control law. The results are demonstrated by numerical examples.
\end{abstract}

\section{INTRODUCTION}

Finite time stable control systems have received considerable interest recently. A Lyapunov function theorey for finitetime stability was proposed in [1] based on a class of Lyapunov functions with constant decay exponent $\alpha \in(0,1)$. This theorem was later extended to more general Lyapunov functions in [2,3]. The finite-time stability theorem for homogeneous systems was first introduced in [4], and has been used as a convenient tool for establishing finite time convergence for multidimensional nonlinear systems. For example, the finite time stability problems for high order nonlinear systems in specific forms were addressed in [5-7] using the homogeneous system approach. The control Lyapunov function also provides a means for the finite-time stabilization of high dimensional nonlinear dynamical systems, and was applied to the finite-time stabilization of homogeneous dynamical systems [8]. Discontinuous stabilizing feedback controllers have also been developed in $[9,10]$, etc. However, discontinuous controllers may cause the excitation of unmodeled high-frequency modes of the system, which is not favorable for

\footnotetext{
${ }^{*}$ This work was done while the author was a graduate student in the School of Aerospace Engineering, Georgia Institute of Technology.
}

practical implementations.

Network control theory has been studied extensively [11, 12]. The network control problems are usually addressed via the Laplacian dynamics, while a variant of this approach based on the edge Laplacian has also been proposed in [13] and [14]. The later approach reveals the roles of different subgraphs and enables the design of network control protocols through the edge agreement.

Reference [15] studied the problem of finite-time convergence to a continuum of equilibria, which is referred to as finitetime semistability, and proposed a smooth state feedback controller architecture for finite-time consensus of network system with the assumption that the information flow between each pair of agents in the network system is always maintained until the consensus between these two agents has been achieved. This assumption is later removed in Ref. [16], which proposed a finitetime output feedback controller for the rendezvous of a system of agents subject to limited range of communication. However, the underlying controller is no longer smooth. In particular, the control inputs could be bang-bang when the rendezvous process is close to completion such that the system states are within a certain distance from the semi-stable equilibrium set.

In this paper, based on current results in finite-time stability and homogeneous system, we will analyze the stability for a special type of nonlinear system, which is related to linear systems via certain nonlinear transformations. It is proved that the finite-time convergence of such systems can be easily achieved by applying homogeneous transformations to produce a negative homogeneity degree. This result is used to design control protocols for a network system containing a spanning tree. It will be shown that the smooth finite-time feedback stabilizing controller proposed in [15] can be generalized to arbitrary connected networks without the full connectedness assumption. As compared to control laws in [10], such a feedback control law is infinitely smooth except for the origin. Finally, numerical examples are 
presented to validate the main theorem of this paper and demonstrate the proposed network control algorithms.

\section{Preliminaries and Notations}

In this paper, we follow the definition of finite-time stability introduced in [1].

Definition 1. Consider a scalar continuous autonomous ordinary differential equation given by

$$
\dot{x}(t)=f(x(t)),
$$

where $f: \mathcal{D} \rightarrow \mathbb{R}_{+}$is a continuous function over some open neighborhood $\mathcal{D} \in \mathbb{R}$ of the origin, and $f(0)=0$. The origin is a finite-time-stable equilibrium of (1) if there exists a neighborhood $\mathcal{B}$ of the origin and a settling time function $T_{f}$ : $\mathcal{B} \backslash\{0\} \rightarrow \mathbb{R}_{+}$such that the following two conditions hold: ( $i$ ) for any $x_{0} \in \mathcal{B} \backslash\{0\}$, the solution $\psi\left(t, x_{0}\right)$ is uniquely defined for all $t \in\left[0, T_{f}\left(x_{0}\right)\right), \psi\left(t, x_{0}\right) \in \mathcal{B}$, and $\lim _{t \rightarrow T_{f}\left(x_{0}\right)} \psi\left(t, x_{0}\right)=0$; (ii) the origin is Lyapunov stable.

It was shown in [1] that $\psi\left(t, x_{0}\right)$ is actually defined on $\mathbb{R}_{+} \times$ $\mathcal{B}$ with $\psi\left(t, x_{0}\right)=0$, for all $t \geq T_{f}\left(x_{0}\right), x \in \mathcal{B}$, and $T_{f}(0)=0$. The origin is said to be a globally finite-time-stable equilibrium if $\mathcal{B}=\mathcal{D}=\mathbb{R}^{n}$.

Next we briefly introduce the notion of homogeneous system [17] and some concepts of graph theory [12], which will be used later in this paper.

Definition 2. A dilation $\Delta_{\lambda}^{\mathbf{r}}: \mathbb{R}^{n} \times \mathbb{R} \rightarrow \mathbb{R}^{n}$ is defined with respect to $x=\left(x_{1}, x_{2}, \ldots, x_{n}\right) \in \mathbb{R}^{n}$ by assigning $n$ positive real numbers $\mathbf{r}=\left(r_{1}, r_{2}, \ldots, r_{n}\right)$ and a positive real number $\lambda$ such that

$$
\Delta_{\lambda}^{\mathbf{r}} x=\left(\lambda^{r_{1}} x_{1}, \ldots, \lambda^{r_{1}} x_{1}\right)
$$

Definition 3. A continuous function $f_{i}: \mathbb{R}^{n} \rightarrow \mathbb{R}$ is homogeneous of degree $l$ if $f_{i}\left(\Delta_{\lambda}^{\mathbf{r}}(x)\right)=\lambda^{l} f_{i}(x)$. A vector field $f$ is homogeneous of degree $m$ with respect to $\Delta_{\lambda}^{\mathbf{r}}$ if each component $f_{i}$ is of degree $m+r_{i}$.

An undirected graph denoted by $\mathcal{G}=(\mathcal{V}, \mathcal{E})$ is composed of a vertex set $\mathcal{V}(\mathcal{G})=\left\{v_{i} ; i=1,2, \ldots, n\right\}$ and an edge set $\mathcal{E}$, which is a particular subset of $\mathcal{V}(\mathcal{G}) \times \mathcal{V}(\mathcal{G})$ which describes the connectedness between vertices [12]. A subgraph of a graph $\mathcal{G}$ is a graph whose vertex and edge sets are subsets of those of $\mathcal{G}$. An orientation of an undirected graph $\mathcal{G}$ is the assignment of directions to its edges.

A path in a directed graph from $v_{i_{1}}$ to $v_{i_{k}}$ is a sequence $v_{i_{1}}, v_{i_{2}}, \ldots, v_{i_{k}} \in \mathcal{V}(\mathcal{G})$ such that $\left(v_{i_{j}}, v_{i_{j+1}}\right) \in \mathcal{E}(\mathcal{G}) . \mathcal{G}=(\mathcal{V}, \mathcal{E})$ has a spanning tree if there exists a vertex which can be connected to all other vertices through paths. This vertex is called a root. A circle is a path that starts and ends at the same vertex.
Following the definitions in Ref. [14], suppose an undirected graph $\mathcal{G}$ contains $n$ vertices and $m$ edges, the $n \times m$ incidence matrix $E(G)$ for an orientation of $\mathcal{G}$ is given by

$$
[E(G)]_{i, k}= \begin{cases}1 & \text { if } v_{i} \text { is the initial node of edge } e_{k} \\ -1 & \text { if } v_{i} \text { is the terminal node of edge } e_{k} \\ 0 & \text { otherwise }\end{cases}
$$

The positive semi-definite $n \times n$ matrix $L(\mathcal{G}) \triangleq E(G) E^{T}(\mathcal{G})$ is called the Laplacian of $\mathcal{G}$, which is a rank-deficient positive semi-definite matrix. The edge Laplacian of $\mathcal{G}$ is defined as $L_{e}(\mathcal{G}):=E(G)^{T} E(G)$, which is a real symmetric $m \times m$ matrix.

\section{Stability Analysis of a Class of Nonlinear System}

Consider the following nonlinear dynamical system:

$$
\dot{x}(t)=A \Phi(x(t)),
$$

where $A \in \mathbb{R}^{n \times n}, x(t)=\left[x_{1}(t), x_{2}(t), \ldots, x_{n}(t)\right]^{T} \in \mathbb{R}^{n}, \Phi:$ $\mathbb{R}^{n} \rightarrow \mathbb{R}^{n}$ be a function on $\mathbb{R}^{n}$ defined by $\Phi(x)=$ $\left[\phi_{1}\left(x_{1}\right), \phi_{2}\left(x_{2}\right), \ldots, \phi_{n}\left(x_{n}\right)\right]^{T}$.

As will be shown, system (3) inherits the stability property of the linear system $\dot{x}=A x$ when $\Phi$ satisfies the following assumptions:

Assumption 1. For all $i=1, \ldots, n, \phi_{i}: \mathbb{R} \rightarrow \mathbb{R} i$ continuous on $\mathbb{R} \backslash\{0\}$, Hölder continuous at the origin, and locally Lipschitz continuous on $(0, \infty), \phi_{i}(0)=0$, and $s \phi_{i}(s)>0$ for all $s \neq 0$. Furthermore, there exists $\varepsilon>0$ and $s_{\varepsilon}>0$ such that $\left|\phi_{i}(s)\right| \geq \varepsilon$ for all $|s|>s_{\varepsilon}$.

Note that no assumption was made on the monotonicity or boundedness of $\phi_{i}$.

Theorem 1. Suppose A is Hurwitz, then system (3) with $\Phi$ satisfying Assumption 1 is globally asymptotically stable.

Proof. Consider the Lyapunov function candidate

$$
V(x)=\sum_{i=1}^{n}\left(\int_{0}^{x_{i}} \phi_{i}(s) \mathrm{ds}\right) .
$$

By the definition of $\Phi$, it is easily verified that $V(x(t)) \geq 0$ for any $x(t) \in \mathbb{R}^{n}, V(0)=0$ and $V(x(t))>0$ for any $x(t) \neq 0, x(t) \in \mathcal{P}$. Also, because $\operatorname{sgn}(\mathrm{s}) \phi_{\mathrm{i}}(\mathrm{s}) \geq \varepsilon>0$ for $|s| \in\left[s_{\varepsilon}, \infty\right), 1 \leq i \leq n$, we have $\int_{0}^{x_{i}} \phi_{i}(s) \mathrm{ds} \rightarrow \infty$ as $x_{i} \rightarrow \infty$, hence $V$ is radially unbounded. Taking the time derivative of $V$, we have

$$
\begin{aligned}
& \dot{V}(x(t))=\sum_{i=1}^{n}\left(\phi_{i}\left(x_{i}(t)\right) \dot{x}_{i}\right) \\
& =\Phi^{T}(x(t)) \dot{x}(t) \\
& =\Phi^{T}(x(t)) A \Phi(x(t)) .
\end{aligned}
$$


Because $A$ is Hurwitz and $\Phi(x) \neq 0$ for $x \neq 0, \dot{V}(x(t))$ is strictly negative for any $x \in \mathbb{R}, x \neq 0$. Hence system (3) is globally asymptotically stable.

According to Theorem 1, system (3) preserves the asymptotic stability of the linear system $\dot{x}(t)=A x(t)$ when $A$ is Hurwitz. As will be shown later in this paper, this conservation of the stability of system (3) can help the design of nonlinear controllers.

When $A$ is symmetric, then a stronger result holds with the following less restrictive assumption on $\Phi$ :

Assumption 2. $\phi_{i}: \mathbb{R}^{n} \rightarrow \mathbb{R}$ is continuous on $\mathbb{R}^{n} \backslash\{0\}$, Hölder continuous at the origin, and locally Lipschitz continuous on $(0, \infty) . \quad \phi_{i}(0)=0, \quad \phi_{i}(z) \neq 0$ and $z_{i} \phi_{i}(z)>0$ for all $z=$ $\left[z_{1}, \ldots, z_{n}\right]^{T} \in \mathbb{R}^{n}$ with $z_{i} \neq 0$. Besides, there exists $\varepsilon>0$ and $s_{\varepsilon}>0$ such that $\left|\phi_{i}(z)\right| \geq \varepsilon$ for any $z$ with $\|z\| \geq s_{\varepsilon}, i=1,2, \ldots, n$.

With the Lyapunov function $V(x)=-x^{T} A^{-1} x$, the following theorem can be proved.

Theorem 2. Suppose $A$ is Hurwitz and symmetric, and $\Phi$ satisfies Assumption 2, then system $\dot{x}=A \Phi(x)$ is globally asymptotically stable.

As an illustrative example for Theorem 1, consider the following two dimensional dynamical system with a constant drift term:

$$
\begin{aligned}
& \dot{x}_{1}=-e^{x_{1}}-3 e^{3 x_{2}}+4 \\
& \dot{x}_{2}=e^{x_{1}}-e^{3 x_{2}}
\end{aligned}
$$

The above dynamical system can be rewritten in the form of (3) with

$$
A=\left[\begin{array}{cc}
-1 & -3 \\
1 & -1
\end{array}\right]
$$

$\Phi(x)=\left[\phi_{1}\left(x_{1}\right) \phi_{2}\left(x_{2}\right)\right]^{T}$, where $\phi_{1}\left(x_{1}\right)=e^{x_{1}}-1$ and $\phi_{2}\left(x_{2}\right)=$ $e^{3 x_{2}}-1$. It can be verified that $\Phi$ satisfies assumption 1 , hence this system is globally asymptotically stable following Theorem 1.

Consider another example of a two dimensional homogeneous system

$$
\begin{aligned}
& \dot{x}_{1}=-3 x_{1}^{3}+2 x_{1}^{2} x_{2}-3 x_{2}^{2} x_{1}+2 x_{2}^{3}, \\
& \dot{x}_{2}=x_{1}^{3}+x_{1}^{2} x_{2}-x_{2}^{2} x_{1}-x_{2}^{3} .
\end{aligned}
$$

The above system can be rewritten as $\dot{x}=A \Phi(x)$, where

$$
A=\left[\begin{array}{cc}
-3 & 1 \\
1 & -1
\end{array}\right]
$$

is Hurwitz and symmetric, and $\Phi(x)=\left[x_{1}^{3}+x_{2}^{2} x_{1}, x_{1}^{2} x_{2}+x_{2}^{2}\right]^{T}$ satisfies Assumption 2, hence, this homogeneous system is globally asymptotically stable according to Theorem 2 .

Now consider a specific class of $\Phi$. Let $\mathbf{r}=$ $\left(r_{1}, r_{2}, \ldots, r_{n}\right)$, where $r_{i}>0$ for $i=1,2, \ldots, n$, and $[x]^{\mathbf{r}}=$ $\left[\operatorname{sgn}\left(x_{1}\right)\left|x_{1}\right|^{r_{1}}, \operatorname{sgn}\left(x_{2}\right)\left|x_{2}\right|^{r_{2}}, \ldots, \operatorname{sgn}\left(x_{n}\right)\left|x_{n}\right|^{r_{n}}\right]^{T}$. If $r_{1}=r_{2}=$ $\cdots=r_{n}=r$, then $[x]^{\mathbf{r}}$ is denoted with $[x]^{r}$.

Corollary 1. Suppose A is Hurwitz. Let $\mathcal{P}$ be a finite index set. $c_{p}>0$, and $\mathbf{r}_{p}=\left(r_{p 1}, r_{p 2}, \ldots, r_{p n}\right), r_{p i}>0$ for any any $p \in \mathcal{P}$. Let $X_{\mathcal{P}}(x)=\sum_{p \in \mathcal{P}} c_{p}[x]^{p_{p}}$. Then the following dynamic system

$$
\dot{x}(t)=A X_{\mathcal{P}}(x(t))
$$

is globally asymptotically stable.

Proof. Let $\Phi(x)=X_{\mathcal{P}}(x(t))$. It is easily verified that $\Phi$ satisfies assumption 1 , hence system (1) is globally asymptotically stable following theorem 1 .

According to [18], if a vector field can be written as the sum of several vector fields, each of them being homogeneous with respect to a fixed dilation, then asymptotic stability of the lowest degree vector field implies local asymptotic stability of the original vector field. System (5) is a special case of such a sum of homogeneous vector fields, and its stability is actually global by Corollary 1.

The following theorem is from [19]:

Theorem 3. Let the Euler vector field $\vee$ be $C^{1}$ and suppose $f=g_{1}+\cdots+g_{k}$, where, for each $i=1, \ldots, k$, the vector field $g_{i}$ is continuous, homogeneous of degree $m_{i}$ with respect to $v$ and $m_{1}<m_{2}<\cdots<m_{k}$. If the origin is a finite-time stable equilibrium under $g_{1}$, then the origin is a finite-time-stable equilibrium under $f$.

From theorem 3, we readily have the following lemma regarding the finite-time stability of system (5):

Lemma 1. Suppose A is Hurwitz, for $p=1, \ldots, P, c_{p}>0,0<$ $\alpha_{1}<\cdots<\alpha_{P}$. if $\dot{x}=A[x]^{\alpha_{1}}$ is finite-time stable, then system (5) with $X_{\mathcal{P}}(x)=\sum_{p=1}^{P} c_{p}[x]^{\alpha_{p}}$ is also finite-time stable.

Proof. For any $\alpha>0$, the vector field $A[x]^{\alpha}$ is homogeneous with the standard dilation $\Delta_{\lambda}(x)=\lambda x$, whose Euler vector field is $C^{1}$. It can be easily verified that the homogeneity degree of $c_{p} A[x]^{\alpha_{p}}$ is $\alpha_{p}-1$ for $p=1, \ldots, P$. Hence, the lemma holds following theorem 3 .

Remark 1. observing that when $A$ is Hurwitz and symmetric, the function $W(x)=-x^{T} A^{-1} x$ is a common Lyapunov function for any systems in the form of (3) with $\Phi$ satisfying either Assumption 1 or assumption2, and arbitrary switching between different systems will not destroy the stability of the overall system. Hence switching control can be designed for taking advantage of the best local convergence speed of different sub-systems. 


\section{Finite-Time Stabilizing State Feedback Control Based on Edge Agreement}

According to Theorem 2 in [4], a homogeneous system is finite-time stable at the origin if and only if the origin is an asymptotically stable point, and the system has a negative homogeneity degree. In this section we use this theorem to design decentralized rendezvous and formation control algorithms based on edge agreement such that the desired control task is achieved within finite time. We also present a method for rendezvous and formation control with bounded control input. Although these protocols are designed for non-weighted directed graph, they can be easily extended to consider weights on edges in the graph without any fundamental change.

Consider a connected network system comprising of $n$ agents $x_{i}(t) \in \mathbb{R}, i=1, \ldots, n$. It is assumed that the graph $\mathcal{G}$ associated with this network system is time-invariant, and contains a spanning tree. We also assume that for any $i, j \in \mathcal{V}, i \neq j$, agent $i$ is able to observe the relative distance $x_{i}-x_{j}$ from agent $j$ if and only if incidence matrix entry $E_{i j}=1$.

The dynamics of the agents is given by

$$
\begin{aligned}
& \dot{x}(t)=u(e(t)), \\
& e(t)=E^{T} x(t),
\end{aligned}
$$

where $x(t)=\left[x_{1}(t), x_{2}(t), \ldots, x_{n}(t)\right]^{T}, e=\left[e_{1}, e_{2}, \ldots, e_{m}\right]^{T}, u$ : $\mathbb{R}^{m} \rightarrow \mathbb{R}^{n}$ is an output feedback control with each of its component $u_{i}$ being a function of those $e_{k}$ with $E(i, k)=1$, for all $i=1, \ldots, n$, such that the control law is ensured to be decentralized. Note that for any $k=1, \ldots, m$, suppose $\left[E_{r}\left(G_{r}\right)\right]_{i, k}=1$ and $\left[E_{r}\left(G_{r}\right)\right]_{j, k}=-1$, i.e., the $k^{\text {th }}$ edge starts from the $i^{\text {th }}$ agent and ends at the $j^{\text {th }}$, then $e_{k}(t)=\left[E_{r}^{T} x(t)\right]_{i}=x_{i}(t)-x_{j}(t)$, which is exactly the measurable relative displacement.

With a linear output feedback control law $u(e(t))=-E e(t)$, system (6) becomes the well-known node agreement dynamics

$$
\dot{x}(t)=-E E^{T} x(t)=-L(\mathcal{G}) x(t) .
$$

With arbitrary initial condition $x(0), x(t)$ in (8) converges to $\gamma \mathbf{1}_{n}$ exponentially for some $\gamma \in \mathbb{R}$, and it takes infinitely long time such that $x(t) \in \operatorname{span}\left\{\mathbf{1}_{\mathrm{n}}\right\}$.

Next, we propose a decentralized output feedback control law such that the agreement between the states can be achieved within finite time, i.e., there exists $T_{f}>0$ such that $x(t) \rightarrow \gamma \mathbf{1}_{n}$ for some $\gamma \in \mathbb{R}$ as $t \rightarrow T_{f}$ and $x(t) \in \operatorname{span}\left\{\mathbf{1}_{\mathrm{n}}\right\}, t \geq T_{f}$. Instead of designing a finite-time stabilizing feedback controller for the node dynamics (6) directly, we will work on the edge dynamics, which is described by the following expression

$$
\dot{e}(t)=-E^{T} \dot{x}(t)=-E^{T} u(e(t))
$$

where $u$ becomes a state feedback law. By definition of $e(t)$, it is clear that when $\mathcal{G}$ is connected, $\|e(t)\| \rightarrow 0$ if and only if $\|x(t)\| \rightarrow \gamma \mathbf{1}_{n}$ for some $\gamma \in \mathbb{R}$, and $\|e(t)\|=0$ if and only if $\|x(t)\| \in \operatorname{span}\left\{\mathbf{1}_{\mathrm{n}}\right\}$. Hence, we can design a control for the edge dynamics such that $e(t)$ converges to the origin in finite time. When $\mathcal{G}$ contains cycles, the components of $e(t)$ are linearly dependent. In order to remove the coupling between the edge states, rewrite the incidence matrix $E$ (possibally after a relabeling of the edges) as [14]:

$$
E=\left[E_{t} E_{c}\right]=E_{t} \mathbf{R}
$$

where $E_{t}$ corresponds to a particular spanning tree in $\mathcal{G}, E_{c}$ describes the remaining edges associated with the cycles in $\mathcal{G}$, $\mathbf{R}=[\mathbf{I} \mathbf{T}]$ and $\mathbf{T}=\left(E_{t}^{T} E_{t}\right)^{-1} E_{t}^{T} E_{c}$. Correspondingly, the edge states can also be partitioned as

$$
e(t)=\left[e_{t}^{T}(t), e_{c}^{T}(t)\right]^{T}=\mathbf{R}^{T} e_{t}(t)
$$

where $e_{t}, e_{c}$ are the column vectors of edges associated with $E_{t}$ and $E_{c}$, respectively.

Consider the feedback control law $u(e(t))=-E \Phi(e(t))$, where $\Phi$ satisfies Assumption 1. It can be easily verified that such a control law is decentralized, and by the assumption on $\Phi$, we may write $\Phi(e(t))=\left[\Phi_{t}\left(e_{t}(t)\right)^{T}, \Phi_{c}\left(e_{c}(t)\right)^{T}\right]^{T}$. Observing that $e_{t}(t)=E_{t}^{T} x(t)$, we have

$$
\begin{aligned}
& \dot{e}_{t}(t)=E_{t}^{T} \dot{x}(t) \\
& =E_{t}^{T} u(e(t)) \\
& =-E_{t}^{T} E \Phi(e(t)) \\
& =-E_{t}^{T}\left[E_{t} E_{c}\right]\left[\Phi_{t}\left(e_{t}(t)\right)^{T} \Phi_{c}\left(e_{c}(t)\right)^{T}\right]^{T} \\
& =-E_{t}^{T} E_{t} \Phi\left(e_{t}(t)\right)-E_{t}^{T} E_{c} \Phi\left(e_{c}(t)\right) \\
& =-L_{e}\left(G_{t}\right) \Phi_{t}\left(e_{t}(t)\right)-E_{t}^{T} E_{c} \Phi_{c}\left(\mathbf{T}^{T} e_{t}(t)\right),
\end{aligned}
$$

where $\mathcal{G}_{t}$ is a subgraph of $\mathcal{G}$ which corresponds to the spanning tree with edges $e_{t}$. Consider first the special case such that $\mathcal{G}=$ $\mathcal{G}_{t}$, i.e., $\mathcal{G}$ is by itself a spanning tree. In this case, the second term in the (11) vanishes. The following theorem shows that finite-time rendezvous can be achieved using a state-feedback controller which is designed based on the spanning tree $\mathcal{G}_{t}$

Theorem 4. Suppose $\mathcal{G}$ contains a spanning tree $\mathcal{G}_{t}$, and let $E_{t}$ be the incidence matrix associated with $\mathcal{G}_{t}$. Let $0<\alpha<1$. With the following feedback control law for (6)

$$
u(x(t))=-E_{t}\left[e_{t}(t)\right]^{\alpha}=-E_{t}\left[E_{t}^{T} x(t)\right]^{\alpha},
$$

there exists $T_{f}>0$ such that $x(t) \rightarrow \operatorname{span} 1$ as $t \rightarrow T_{f}$, and $x(t) \in$ $\operatorname{span} 1$ for $t \geq T_{f}$.

Proof. Let $L_{e}\left(G_{t}\right)=E_{t}^{T} E_{t}$ be the edge Laplacian for $\mathcal{G}_{t}$. Then $L_{e}\left(G_{t}\right)$ is strictly positive definite. Consider the reduced edge dynamics with the control in (12)

$$
\dot{e}_{t}(t)=-L_{e}\left(\mathcal{G}_{t}\right)\left[e_{t}\right]^{\alpha}
$$


Because $-L_{e}\left(G_{t}\right)$ is Hurwitz, system (13) is globally asymptotically stable following Theorem 1 . Note that (13) is homogeneous with respect to the standard dilation $\Delta_{\lambda}(e)=\lambda e$. Let $g\left(e_{t}\right)=-L_{e}\left(\mathcal{G}_{t}\right)\left[e_{t}\right]^{\alpha}$. Apply $\Delta_{\lambda}$ to $g$, we have

$$
\begin{aligned}
g\left(\Delta_{\lambda}\left(e_{t}\right)\right) & =-L_{e}\left(\mathcal{G}_{t}\right)\left[\lambda e_{t}\right]^{\alpha} \\
& =-\lambda^{\alpha} L_{e}\left(G_{t}\right)\left[e_{t}\right]^{\alpha} \\
& =\lambda^{1+\alpha-1} g\left(e_{t}\right) .
\end{aligned}
$$

Hence, system (13) has a homogeneity degree of $\alpha-1$. By Theorem 2 in [4], there exists a real number $T_{f}>0$ such that $e_{t}(t) \rightarrow 0$ as $t \rightarrow T_{f}$, and $e_{t}(t)=0$ for $t \geq T_{f}$. Because $e_{t}(t)=0$ if and only if $x(t) \in \operatorname{span} 1$, the proof is complete.

As an extension to Theorem 12, consider the case when the undirected graph $\mathcal{G}$ of the network topology is connected, then $\mathcal{G} \supset \mathcal{G}_{r}$, but $\mathcal{G} \neq \mathcal{G}_{r}$. The dimension of the incidence matrix $E\left(\mathcal{G}_{r}\right)$ is $n \times(n-1)$, and the edges of the spanning tree is determined by $e_{r}(t)=E^{T}\left(\mathcal{G}_{r}\right) x(t)$, which is a $n-1$ dimensional vector measuring the relative displacements between certain agents in the network. Because the spanning tree of a connected graph is not necessarily unique, so is the selection of the reduced edges. The following convergence analysis holds with any choice of $e_{r}$.

Consider the rendezvous problem in the form of (6) with nonlinear feedback control $u(x(t))=-E \Phi(e(t)))$, where $e(t)=$ $E^{T}(\mathcal{G}) x(t)$. Assuming that the topology of the network is invariant, $E(\mathcal{G})$ does not change with respect to time, thus we have $\dot{e}(t)=E^{T}(\mathcal{G}) \dot{x}(t)$. The dynamics of the edges $e(t)$ is described by

$$
\dot{e}(t)=-E^{T}(\mathcal{G}) E(\mathcal{G}) \Phi(e(t))=-L_{e}(\mathcal{G}) \Phi(e(t)) .
$$

Because $\mathcal{G}$ itself is not a spanning tree, $L_{e}(\mathcal{G})$ is only positive semi-definite, hence theorem 1 can not be applied for the convergence analysis of (14). Besides, the components of $e(t)$ are not linearly independent, which is inconvenient for studying the convergence of $e(t)$ to the origin.

When $\mathcal{G}$ contains, but is not, a spanning tree, the stability of the reduced edge dynamical system is given by the following theorem

Theorem 5. Let $\mathcal{G}$ be the undirected graph of a network system which is connected, and $\Phi$ satisfies assumption 1. Then the rendezvous problem in the form of (6) is solved with feedback control $u(x(t))=-E \Phi(e(t))$.

Proof. Because $\mathcal{G}$ is connected, it contains a spanning tree $\mathcal{G}_{r}$. Let

$$
\begin{aligned}
\delta(x(t)) & =x(t)-\frac{1}{N} \mathbf{1}^{T} x(t) \mathbf{1} \\
& =\left(\mathbf{I}-\frac{1}{N} \mathbf{1 1}^{T}\right) x(t),
\end{aligned}
$$

which is the component of the $x(t)$ in the subspace which is orthogonal to $\operatorname{span}\{\mathbf{1}\}$ (which is also referred to as the disagreement subspace). Noting that the rendezvous problem is achieved if and only if $\delta(x(t))$ vanishes. Let

$$
\zeta\left(e_{r}(t)\right)=\delta(x(t))
$$

$\zeta$ is indeed a function of $e(t)$. To see this, notice that the $i^{\text {th }}$ component of $\zeta$ :

$$
\zeta_{i}=\left[\left(\mathbf{I}-\frac{1}{N} \mathbf{1 1}^{T}\right) x(t)\right]_{i}=\frac{1}{N} \sum_{j=1 ; j \neq i}^{n}\left(x_{i}(t)-x_{j}(t)\right)
$$

Because $\mathcal{G}$ contains a spanning tree $\mathcal{G}_{r}$, any agent is connected to the route of $\mathcal{G}_{r}$ by certain path consisting of edges in $\mathcal{G}_{r}$, then $x_{i}(t)-x_{j}(t)$ can be written as a linear combination of the edges in $\mathcal{G}_{r}$ which compose the two pathes connecting the agents to the root, therefore, there exists a matrix $Q \in \mathbb{R}^{n \times(n-1)}$ such that

$$
\zeta\left(e_{r}(t)\right)=\delta(x(t))=Q e_{r}(t) .
$$

Consider the following Lyapunov function candidate for the reduced edge dynamics (11)

$$
V\left(e_{r}(t)\right)=\frac{1}{2} \zeta^{T}\left(e_{r}(t)\right) \zeta\left(e_{r}(t)\right)
$$

Clearly $V\left(e_{r}(t)\right) \geq 0$, and $V\left(e_{r}(t)\right)=0 \Leftrightarrow \zeta\left(e_{r}(t)\right)=0 \Leftrightarrow$ $\delta(x(t))=0 \Leftrightarrow x(t) \in \operatorname{span}\{\mathbf{1}\} \Leftrightarrow e_{r}(t)=0$, where the last equivalence relation holds because $\mathcal{G}$ contains a spanning tree, and this implies that $\mathcal{N}\{V\}=0$. Since $V(e(t))=1 / 2\|Q e(t)\|^{2}$, we have that $\mathcal{N}\{Q\}=\mathcal{N}\{V\}=0$, which means that $Q^{T} Q$ is a positive definite matrix, and $V$ is radially unbounded, hence (17) is indeed a Lyapunov function candidate. Taking the time derivative of $V$, we have

$$
\begin{aligned}
\dot{V}\left(e_{r}(t)\right) & =\delta^{T}(x(t)) \dot{\delta}(x(t)) \\
& =x(t)^{T}\left(\mathbf{I}-\frac{1}{N} \mathbf{1 1}^{T}\right)^{2} \dot{x}(t) \\
& =x(t)^{T}\left(\mathbf{I}-\frac{1}{N} \mathbf{1 1}^{T}\right)^{2} u(x(t)) \\
& =-x(t)^{T}\left(\mathbf{I}-\frac{1}{N} \mathbf{1 1}^{T}\right)^{2} E \Phi(e(t))
\end{aligned}
$$

Because $\mathbf{1} \in \mathcal{N}\left(E^{T}\right), \mathbf{1}^{T} E=\left(E^{T} \mathbf{1}\right)^{T}=0$, the above expression 
can be further simplified to

$$
\begin{aligned}
\dot{V}\left(e_{r}(t)\right) & =-x(t)^{T} E \Phi(e(t)) \\
& =-\left(E^{T} x(t)\right)^{T} \Phi(e(t)) \\
& =-e^{T}(t) \Phi(e(t)) \\
& \leq-e_{r}^{T}(t) \Phi\left(e_{r}(t)\right) \\
& \leq 0
\end{aligned}
$$

By definition of $\Phi, e_{r}^{T}(t) \Phi\left(e_{r}(t)\right)=0$ if and only if $e_{r}(t)=0$, hence the reduced edge dynamics (11) is asymptotically stable, which implies that $x(t) \rightarrow \gamma \mathbf{1}$ for some $\gamma \in \mathbb{R}$ as $t \rightarrow+\infty$.

Theorem 5 explores the solution of network control problem in the context of nonlinear feedback. It shows that consensus can be achieved so long as the nonlinear feedback control law satisfies assumption 1. The commonly used linear feedback for network consensus is a special case of theorem 5 with $\Phi(e(t))=$ $e(t)$.

The following theorem provides a solution to the finite-time rendezvous problem:

Theorem 6. Assuming that $\mathcal{G}$ is connected, and $\Phi(x)=$ $\sum_{p=1}^{P} c_{p}[x]^{\alpha_{p}}$, where $c_{p}>0, P \geq 1,0<\alpha_{1}<\cdots<\alpha_{P}$, and $\alpha_{1}<1$. Then with the feedback control $u(x(t))=-\Phi(E x(t))$, there exists a real number $T_{f}>0$ such that $x(t) \rightarrow \operatorname{span}\{\mathbf{1}\}$ as $t \rightarrow T_{f}$ and $x(t) \in \operatorname{span}\{\mathbf{1}\}$ for $t \geq T_{f}$.

Noticing that the right hand side of equation (11) is homogeneous when $\Phi(x)=[x]^{\alpha}$ for any $\alpha>0$, the proof is similar to that of Theorem 4 , hence is omitted.

\subsection{A Finite-time Formation Control Algorithm}

Lemma 2. Let $N(i)=\left\{j \mid(i, j) \in \mathcal{E}\left(\mathcal{G}_{r}\right)\right\}$. With the same assumptions as in theorem 5, the following protocol solves the finite-time formation problem.

$$
\begin{aligned}
& u_{i}=-\sum_{j \in N(i)}\left[\operatorname{sgn}\left(\left(x_{i}-x_{j}\right)-\left(h_{i}-h_{j}\right)\right)\right. \\
& \left.\left|\left(x_{i}-x_{j}\right)-\left(h_{i}-h_{j}\right)\right|^{\alpha}\right]
\end{aligned}
$$

for $i=1,2, \ldots, n, 0<\alpha<1$.

Proof. Let $z_{i}=x_{i}-h_{i}, z=\left[z_{1}, z_{2}, \ldots, z_{n}\right]^{T}$, then $x_{i}-x_{j}=h_{i}-h_{j}$ if and only if $z_{i}=z_{j}$, i.e., the formation is achieved if and only if $z \rightarrow \operatorname{span}\left\{\mathbf{1}_{\mathrm{n}}\right\}$. According to theorem 6 , the system

$$
\dot{z}_{i}(t)=-\sum_{j \in N(i)} \operatorname{sgn}\left(z_{i}-z_{j}\right)\left|z_{i}-z_{j}\right|^{\alpha}
$$

for $i=1,2, \ldots, n . z \rightarrow \operatorname{span}\left\{\mathbf{1}_{\mathrm{n}}\right\}$ in finite time, hence with the control protocol (18), the desired formation is achieved in finite time.

\subsection{Network Control with Bounded Control Input}

It is usually assumed that the control in (6) is unbounded during rendezvous or formation, yet in practice the maximum allowable control of each agent is limited, and needs to be addressed in the controller design. Consider the consensus equation with control bounds

$$
\dot{x}(t)=u_{i}(x(t))
$$

where $\left|u_{i}(x(t))\right|<U, \quad i=1, \ldots, n$. Without loss of generality, assume that the graph of the network $\mathcal{G}$ contains a spanning tree $\mathcal{G}_{r}$. The rendezvous problem is solved using (19) with the following bounded control

$$
u_{i}(x(t))=-\frac{U}{\mu(N(i))} \sum_{j \in N(i)} \tanh \left(x_{i}-x_{j}\right)
$$

where $\mu$ is the counting measure. It is easily verified that $\left|u_{i}(x(t))\right|<U$ for $i=1, \ldots, n$. Theorem 5 guarantees that with the above control, the agents achieve consensus asymptotically. Similar control protocol can also be design for the formation control problem with bounded control input.

\section{Numerical Examples}

In this section, we first illustrate the previously proposed finite time (FT) and the bounded control input (BCI) rendezvous algorithms proposed with a randomly generated undirected graph containing 12 vertices and 16 edges. The finite time rendezvous algorithm and the bounded control input rendezvous algorithm start for an arbitrary initial condition. The state and control history are compared with the commonly used asymptotic convergent rendezvous algorithm using linear output feedback in Fig. 1 and 2.
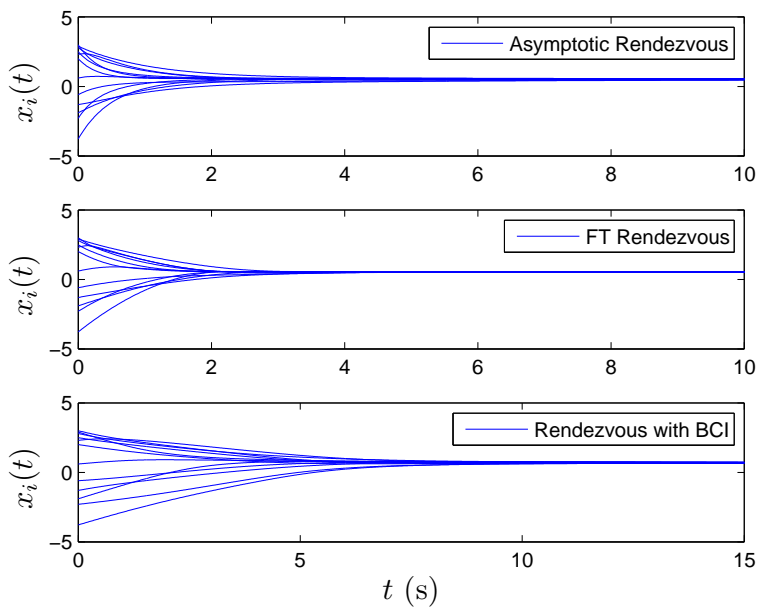

Figure 1. Rendezvous state history. 

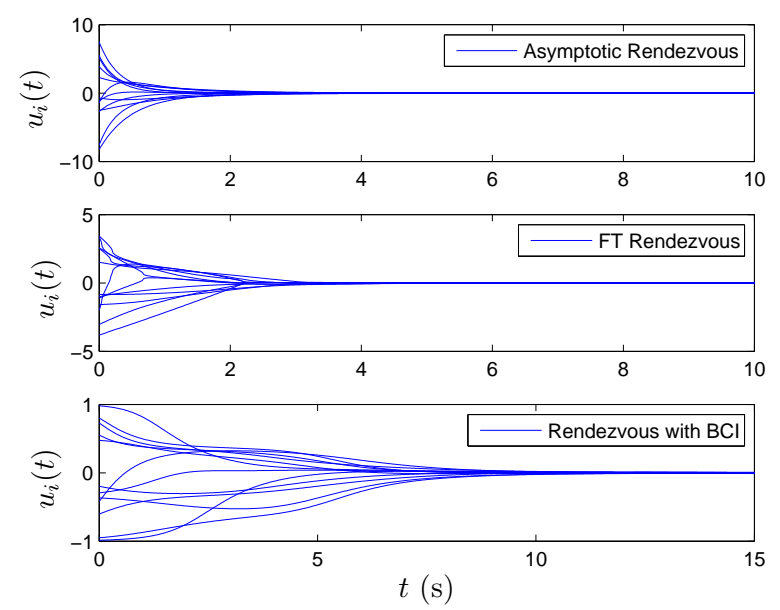

Figure 2. Rendezvous control history.

Note that with the finite time rendezvous protocols, the agents may no longer converge to the centroid of the initial configuration compared with the commonly used linear feedback rendezvous control.

Next, we consider a formation control problem in the 2D plane. The desired formation is composed of one hexagon and three pentagons with equal side length, as shown in Fig.3. The agents are required to be located at the vortices of the polygons at the end of formation. The communication between the agents is also shown in the same figure. The network corresponds to a undirected graph containing 15 vortices and 24 edges. The trajectory of the agents during the formation and the final deployment are shown in Fig. 4. The state histories of the $x$ coordinate position errors for different formation control protocols are shown in Fig. 5. The state histories of the $y$ coordinate errors are similar, hence are omitted.

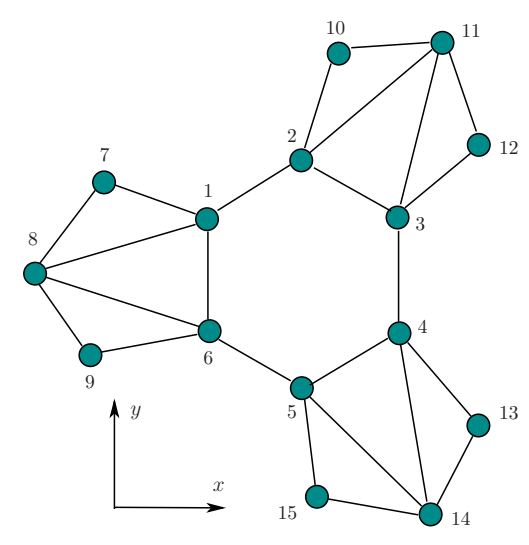

Figure 3. Desired formation and network topology.

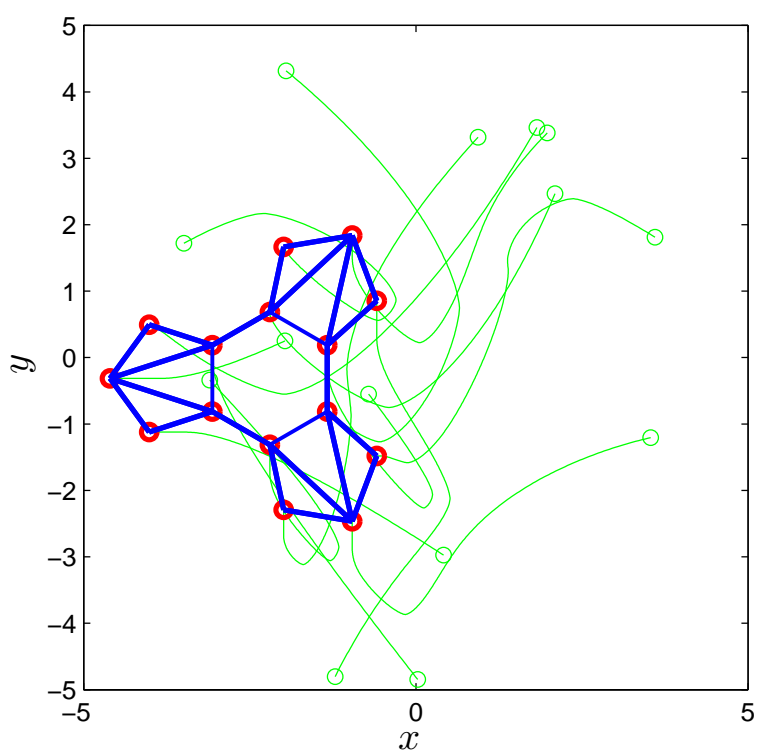

Figure 4. Finite-time formation.
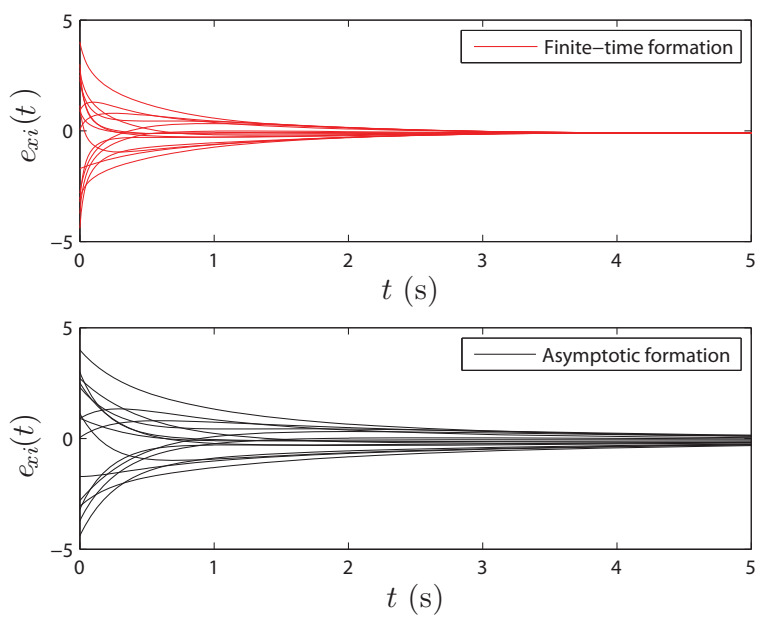

Figure 5. Finite-time formation $x$ coordinate error histories.

\section{Conclusions}

We have shown that the stability of linear system is preserved by a class of nonlinear transformation. This property can be directly applied to the design of finite-time consensus algorithms based on the spanning tree. For an arbitrary connected undirected network, it is shown that finite-time consensus is still guaranteed with the same class of nonlinear feedback of the relative displacements between the agents, which provides more flexibility for the design of decentralized network consensus protocols.

\section{REFERENCES}

[1] Bhat, S. P., and Bernstein, D. S., 2000. "Finite-time stability of continuous autonomous systems". SIAM J. Control Optim., 38(3), pp. 751-766. 
[2] Moulay, E., and Perruquettic, W., 2008. "Finite time stability conditions for non-autonomous continuous systems". International Journal of Control, 81(5), May, pp. 797 803.

[3] Moulay, E., Dambrine, M., Yeganefar, N., and Perruquettic, W., 2008. "Finite-time stability and stabilization of time-delay systems". Systems \& Control Letters, 57(7), Jul, pp. 561-566.

[4] Bhat, S. P., and Bernstein, D. S., 1997. "Finite-time stability of homogeneous systems". In Proceedings of the American Control Conference.

[5] Hong, Y., 2002. "Finite-time stabilization and stabilizability of a class of controllable systems". Systems \& Control Letters, 46, pp. 231-236.

[6] Nakamura, H., Yamashita, Y., and Nishitani, H., 2003. "Finite-time stabilization of the high-order chained system". In Proceedings 01 the American Control Conference.

[7] Li, J., and Qian, C., 2006. "Global finite-time stabilization by dynamic output feedback for a class of continuous nonlinear systems". IEEE Transactions on Automatic Control, 51(5), May, pp. 879-884.

[8] Nersesov, S. G., Haddad, W. M., and Hui, Q., 2007. “Finitetime stabilization of nonlinear dynamical systems via control vector lyapunov functions". In Proceedings of the 2007 American Control Conference, pp. 4810-4816.

[9] Ryan, E. P., 1979. "Singular optimal controls for secondorder saturating systems". International Journal of Control, 30, p. 549564.

[10] Ryan, E. P., 1991. "Finite-time stabilization of uncertain nonlinear planar systems". Dynamics and control, 1, p. 83C 94.

[11] Olfati-Saber, R., Fax, J. A., and Murray, R. M., 2007. "Consensus and cooperation in networked multi-agent systems". In Proceedings of the IEEE, Vol. 95, pp. 215 - 233.

[12] Mesbahi, M., and Egerstedt, M., 2010. Graph-Theoretic Methods for Multiagent Networks. Princeton University Press, Princeton, NJ.

[13] Zelazo, D., Rahmani, A., and Mesbahi, M., 2007. "Agreement via the edge laplacian". In Proceedings of the 46th IEEE Conference on Decsion and Control, pp. 2309-2314.

[14] Zelazo, D., Rahmani, A., Sandhu, J., and Mesbahi, M., 2008. "Decentralized formation control via the edge laplacian". In Proceedings of American Control Conference, pp. 783-788.

[15] Hui, Q., Haddad, W. M., and Bhat, S., 2008. "Finite-time semistability and consensus for nonlinear dynamical networks". IEEE Transactions on Automatic Control, 53(8), Sep, pp. 1887-1900.

[16] Hui, Q., 2011. "Finite-time rendezvous algorithms for mobile autonomous agents". IEEE Transactions on Automatic Control, 56(1), pp. 207-211.

[17] Rosier, L., 1992. "Homogeneous lyapunov function for homogeneous continuous vector field". Systems \& Control Letters, 19, pp. 467-473.

[18] Hermes, H., 1991. Differential equations: stability and control, Vol. 127 of Lecture Notes in Pure and Applied Mathematics. Marcel Dekker, ch. Homogeneous coordinates and continuous asymptotically stabilizing feedback controls, pp. 249-260.

[19] Bhat, S. P., and Bernstein, D. S., 2005. "Geometric homogeneity with applications to finite-time stability". Mathematics of Control, Signals, and Systems, 17, pp. 101-127. 